\title{
Microscopic realization of the Kerr/CFT correspondence
}

\author{
Monica Guica $^{a}$ and Andrew Strominger ${ }^{b}$ \\ ${ }^{a}$ Institut de Physique Théorique, CEA Saclay, \\ CNRS-URA 2306, 91191 Gif sur Yvette, France \\ ${ }^{b}$ Center for the Fundamental Laws of Nature, \\ Jefferson Physical Laboratory, Harvard University, \\ Cambridge, MA 02138, U.S.A. \\ E-mail: monica.guica@cea.fr, strominger@physics.harvard.edu
}

Abstract: Supersymmetric M/string compactifications to five dimensions contain BPS black string solutions with magnetic graviphoton charge $P$ and near-horizon geometries which are quotients of $A d S_{3} \times S^{2}$. The holographic duals are typically known 2D CFTs with central charges $c_{L}=c_{R}=6 P^{3}$ for large $P$. These same 5D compactifications also contain non-BPS but extreme Kerr-Newman black hole solutions with $\mathrm{SU}(2)_{L}$ spin $J_{L}$ and electric graviphoton charge $Q$ obeying $Q^{3} \leq J_{L}^{2}$. It is shown that in the maximally charged limit $Q^{3} \rightarrow J_{L}^{2}$, the near-horizon geometry coincides precisely with the right-moving temperature $T_{R}=0$ limit of the black string with magnetic charge $P=J_{L}^{1 / 3}$. The known dual of the latter is identified as the $c_{L}=c_{R}=6 J_{L}$ CFT predicted by the Kerr/CFT correspondence. Moreover, at linear order away from maximality, one finds a $T_{R} \neq 0$ quotient of the $A d S_{3}$ factor of the black string solution and the associated thermal CFT entropy reproduces the linearly sub-maximal Kerr-Newman entropy. Beyond linear order, for general $Q^{3}<J_{L}^{2}$, one has a finite-temperature quotient of a warped deformation of the magnetic string geometry. The corresponding dual deformation of the magnetic string CFT potentially supplies, for the general case, the $c_{L}=c_{R}=6 J_{L} \mathrm{CFT}$ predicted by Kerr $/ \mathrm{CFT}$.

KeYwords: Gauge-gravity correspondence, Black Holes in String Theory, AdS-CFT Correspondence, Conformal Field Models in String Theory

ARXIV EPRINT: 1009.5039 


\section{Contents}

1 Introduction $\quad 1$

2 The conjecture 3

3 Geometry 5

3.1 Full solution 5

3.2 Six-dimensional lift 6

3.3 Extremality

3.4 Near-horizon limit 8

$\begin{array}{llr}4 & \text { The asymptotic symmetry group } & 9\end{array}$

5 CFT interpretation $\quad 10$

5.1 The maximal case 11

$\begin{array}{ll}5.2 & \text { Explicit brane construction } \\ 5.3 & 12\end{array}$

$\begin{array}{lll}5.3 & \text { Linear deformations } & 13\end{array}$

$\begin{array}{lll}5.4 & \text { Finite deformations } & 14\end{array}$

$\begin{array}{ll}\text { A Long vs. short string pictures } & 15\end{array}$

\section{Introduction}

A complete accounting of the quantum microstates of a black hole requires a complete quantum theory of gravity. Such a complete accounting was obtained for certain supersymmetric black holes [1] by using string theory to identify their microstates with those of a dual two-dimensional conformal field theory (2D CFT). Later it was realized [2] that notable features of the construction - in particular, matching the universality of the $2 \mathrm{D}$ CFT Cardy formula to the universality of the Bekenstein-Hawking area law - followed largely from a careful analysis [3] of the properties of the diffeomorphism group in the $A d S_{3}$ region near the horizon. This implies that the leading order agreement of the microscopic and macroscopic pictures does not require any fine details of string theory. Rather, it must follow a similar pattern for black holes with $A d S_{3}$ near-horizon regions in any consistent ultraviolet completion of quantum gravity. ${ }^{1}$ This is of course in accord with the expectation that universal long-distance phenomena should not depend on the details of short-distance microphysics. It has nevertheless been essential to our understanding to have the specific microscopic examples in string theory [1] in which the general arguments of [2] are given a concrete and fully self-consistent realization.

\footnotetext{
${ }^{1}$ Of course it is a logical possibility that string theory is the only such completion.
} 
Recently, a general analysis of the near-horizon diffeomorphisms of astrophysical Kerr black holes with near-maximal spins, patterned after [2,3], has similarly indicated that they are dual to 2D CFTs [4], but so far there has been no proposed string theoretic realization of the duality. Evidence for the conjectured Kerr/CFT correspondence is given by the agreement of the Cardy formula with the area law, the CFT correlators with near-superradiant Press-Teukolsky greybody factors [5-8], and many other analyses [9-33]. However, there are several puzzling features ${ }^{2}$ which are hard to understand without a concrete microscopic construction. It is the purpose of this paper to fill this gap by proposing such a construction for the general extreme five-dimensional Kerr-Newman black hole embedded as a solution of string theory.

The basic idea is as follows. Supersymmetric M/string compactifications to five dimensions have BPS string solutions with magnetic graviphoton charge $P$ and a near-horizon $A d S_{3} \times S^{2}$ geometry. Typically, the dual CFT is explicitly known from a brane construction and has central charges $c_{L}=c_{R}=6 P^{3}$ for large $P$. We will refer to it as the " $P^{3}$ CFT". For the special case of M-theory on a Calabi-Yau, the $P^{3}$ CFT is the worldvolume theory of an M5-brane wrapping the 4-cycle associated to $P$, and is often referred to as the MSW CFT [34]. We shall be primarily interested in the case where the Calabi-Yau contains a torus factor and there is an uplift from five to six dimensions using the graviphoton fiber. The total space of the graviphoton fiber over the near-horizon $A d S_{3} \times S^{2}$ geometry is then a quotient of $A d S_{3} \times S^{3}[35,36]$.

The same compactifications also contain non-supersymmetric but extreme Kerr-Newman black holes with $\mathrm{SU}(2)_{L}$ spin $J_{L}$ and electric graviphoton charge obeying $Q^{3} \leq J_{L}^{2}{ }^{3}$ These all have a near-horizon $A d S_{2} \times S^{3}$ geometry where the $S^{3}$ is fibered over the $A d S_{2}$ and squashed, known as the 5D NHEK-Newman geometry. Including the graviphoton fiber we get a warped/squashed $A d S_{3} \times S^{3}$ geometry.

The key observation is that in the maximally charged limit $Q^{3} \rightarrow J_{L}^{2}$, the squashing and warping go away and the near-horizon NHEK-Newman geometry coincides exactly with the near-horizon geometry of the charge $P=J_{L}^{1 / 3}$ magnetic string, ${ }^{4}$ with a quotient which corresponds to a right-moving temperature $T_{R}=0$ on the string. Hence the maximal KerrNewman black hole is also dual to the $P^{3} \mathrm{CFT} !^{5}$ The values $c_{L}=c_{R}=6 P^{3}=6 J_{L}$ for this CFT agree with the predictions of the Kerr/CFT correspondence. The full asymptotically flat black hole and black string geometries are of course very different. The gluing of the near-horizon region into the asymptotically flat region of the black hole geometry differs from that of the black string geometry by an exchange of the fiber and spatial angular coordinate. This results in very different dictionaries relating the spacetime and $P^{3} \mathrm{CFT}$ quantum numbers.

\footnotetext{
${ }^{2}$ For example the appearance of complex conformal weights or the apparent lack of boundary conditions admitting two Virasoros.

${ }^{3} Q=0$ is just $5 \mathrm{D}$ extreme Kerr, and we shall refer to the $Q^{3}=J_{L}^{2}$ limit as the maximal case.

${ }^{4}$ In order for this to make sense, $J_{L}$ must be the cube of an integer. However this is not a restriction in the semiclassical limit of interest here because, restoring the factors of $\hbar$, the spacing $\Delta J_{L}$ between two allowed values of $J_{L}$ goes as $\Delta J_{L} \sim \hbar^{1 / 3} J_{L}^{2 / 3}$. Hence the allowed values are effectively continuous for $\hbar \rightarrow 0$.

${ }^{5} \mathrm{~A}$ very similar relation to the supersymmetric $J_{L}^{2}<Q^{3}$ BMPV black holes for $J_{L}^{2} \rightarrow Q^{3}$ was found in [36].
} 
Once we have identified the holographically dual CFT for maximal Kerr-Newman, the duals for the sub-maximal extreme Kerr-Newman can be found by matching the deformation of the bulk geometry to one of the boundary CFT. At linear order, the only effect relevant for the entropy computation is that the action of the quotient is changed in a manner that corresponds to a non-zero right-moving temperature $T_{R}$. Inserting this value of $T_{R}$ into Cardy's formula perfectly reproduces the area law, in accord with the conjectured Kerr/CFT correspondence. At second order, a new effect is relevant: the $A d S_{3}$ factor becomes warped due to a non-zero "self-dual" electric graviphoton field which preserves $\mathrm{SL}(2, \mathbb{R})_{L}$ but breaks $\mathrm{SL}(2, \mathbb{R})_{R}$ to $\mathrm{U}(1)$. In principle this should be describable directly as some kind of continuous deformation of the dual $P^{3}$ CFT and quantum state, but it is beyond the aspirations of the present paper to do so. The Kerr/CFT analysis indicates that the deformed theory exists, and is a CFT whose Cardy entropy matches the area law. However, now that we have embedded Kerr/CFT duality into string theory, standard string theory techniques should be applicable to work out the detailed form of the holographic dual and microscopically verify the Kerr/CFT picture. We will return to the central problem of understanding the deformations beyond linear order away from maximality from the CFT side elsewhere.

An important feature of the finitely-deformed geometry is that it is no longer possible (at least in the most naive fashion) to glue on an asymptotically flat region suitable for a $5 \mathrm{D}$ black string. Gluing on the asymptotically flat Kerr-Newman region requires a specific algebraic relation correlating the temperature $T_{R}$ with the warping factors. This gluing constraint is essential for the consistency of the whole picture: otherwise the number of CFT and black hole parameters would fail to match.

This paper is organized as follows. In section 2 we state the conjecture. In section 3 we review the geometry and all necessary formulae for the extreme 5D Kerr-Newman solution, as well as its 6D lift. Section 4 reviews the Kerr/CFT analysis which leads to an asymptotic symmetry group gnerated by a $c=6 J_{L}$ Virasoro. In section 5 , it is shown that for maximal spin the bulk geometry locally acquires an $\mathrm{AdS}_{3}$ factor and we can thereby identify the boundary $c=6 J_{L}$ CFT dual. An explicit example of a brane construction in which the microscopic dual is the MSW string arising from 3 intersecting stacks of P M5 branes in a $T^{6}$ compactification of $\mathrm{M}$ theory is worked out in detail. Linear deformations from maximality are analyzed, agreement between the bulk and boundary properties is demonstrated and the general non-maximal case is discussed.

Some comments on the existence of different dual CFTs related to the long and short string pictures are made in an appendix.

\section{The conjecture}

Five-dimensional supergravity has black hole solutions characterized by a mass $M$, halfintegral $\mathrm{SU}(2)_{L} \times \mathrm{SU}(2)_{R}$ spins $J_{L}$ and $J_{R}$ and graviphoton charge $Q$. In this paper we consider a two-parameter family of non-supersymmetric extremal spinning black holes 
characterized by

$$
J_{R}=0, \quad Q^{3} \leq J_{L}^{2}, \quad\left(\frac{M}{3}-\frac{Q}{2}\right)^{2}\left(\frac{2 M}{3}+2 Q\right)=J_{L}^{2} .
$$

The Bekenstein-Hawking area law yields the entropy

$$
S_{B H}=2 \pi \sqrt{J_{L}^{2}-Q^{3}}
$$

These black holes are distinct from the supersymmetric BMPV black holes which can have the same spin but must have $Q^{3} \geq J_{L}^{2}$ and $M=3 Q$.

The theory also contains supersymmetric black string solutions characterized by magnetic graviphoton charge $P$ and a near-horizon $A d S_{3} \times S^{2}$ geometry. When this is embedded in $\mathrm{M} /$ string theory the dual $2 \mathrm{D}$ " $P^{3}$ " $\mathrm{CFT}$ is known in many cases [34] and has left and right central charges for large $P$

$$
c_{L}=c_{R}=6 P^{3} \text {. }
$$

The conjecture is that the extremal black holes obeying (2.1) with spin

$$
J_{L}=P^{3}
$$

are dual to a deformation of the known $P^{3} \mathrm{CFT}$ at temperatures

$$
T_{L}=0, \quad T_{R}=\frac{\sqrt{1-\frac{Q^{3}}{J_{L}^{2}}}}{\pi} .
$$

The CFT is deformed by the addition of certain operators to the action, described herein in the bulk picture in terms of a continuous warping of the near-horizon $A d S_{3} \times S^{2}$ geometry. Note that $c_{R}=c_{L}=6 J_{L}$ as expected in the Kerr/CFT correspondence.

Our conjecture is best-motivated in the extended supersymmetric cases of M-theory on $T^{6}$ or $K 3 \times T^{2}$. However, one may consider a more general compactification of $\mathrm{M}$ theory on a Calabi-Yau $X$, which give rise to $\mathcal{N}=25 \mathrm{~d}$ supergravity coupled to $n-1$ vector multiplets. The vector moduli space of the compactification is parametrised by scalars $t^{A}$, $A=1, \ldots n$, which satisfy

$$
D_{A B C} t^{A} t^{B} t^{C}=1
$$

where $6 D_{A B C}$ are the triple self-intersection numbers of the four-cycles of $X$. The black hole is charaterised by $n$ electric charges $q_{A}$ and the angular momentum $J_{L}$. At the horzion, the moduli must take attractor values which obey

$$
q_{A}=3 Q D_{A B C} t^{B} t^{C}
$$

where $Q$ is the graviphoton charge. For this setup, our proposal is that the black hole is described by a deformation of the MSW CFT associated to wrapping an M5-brane on the cycle

$$
p^{A}=J_{L}^{1 / 3} t^{A}, \quad D_{A B C} p^{A} p^{B} p^{C}=J_{L}
$$


This MSW CFT has central charges

$$
c_{L}=c_{R}=6 D_{A B C} p^{A} p^{B} p^{C}=6 J_{L}
$$

plus corrections which are subleading for large $J_{L}$. The extremal black hole is then conjecturally dual to a thermal state in the deformed CFT in which right-movers are excited to the temperature $T_{R}$ given in (2.5).

Note that the M5-brane wraps the four-cycle whose self-intersection is the two-cycle associated to the M2 charge carried by the black hole in the maximal case $Q^{3}=J_{L}^{2}$, and hence has the same attractor point (2.8). Of course not every two-cycle is the self intersection of a four-cycle: our conjecture in its present form pertains only to such cases.

\section{Geometry}

Five dimensional supergravity contains the universal gravity-graviphoton sector

$$
S_{5}=\frac{1}{4 \pi^{2}} \int d^{5} x\left(\sqrt{-g}\left(R-\frac{3}{4} F^{2}\right)+\frac{1}{4} \epsilon^{a b c d e} A_{a} F_{b c} F_{d e}\right) .
$$

We choose conventions with $G_{5}=\frac{\pi}{4}, \epsilon^{\operatorname{tr} \theta \psi \phi}=1$ and $\frac{1}{2 \pi} \int F \in \mathbb{Z}$. Dirac quantization then implies the electric charge $Q$ defined here as $Q \equiv \frac{1}{4 \pi^{2}} \int * F$ obeys $3 Q \in \mathbb{Z}$.

\subsection{Full solution}

We consider a five-dimensional charged rotating black hole solution parameterized by the mass $\mathrm{M}, \mathrm{SU}(2)_{L}$ spin $J_{L}$ and graviphoton charge $Q$. These can be traded for another set of three parameters $a, M_{0}$ and $\delta$ via the relations

$$
\begin{aligned}
M & =\frac{3 M_{0}}{2} \cosh 2 \delta \\
J_{L} & =a M_{0}\left(c^{3}+s^{3}\right), \\
Q & =M_{0} s c
\end{aligned}
$$

where $c=\cosh \delta$ and $s=\sinh \delta$. The black hole solution reads [37-39]

$$
\begin{aligned}
d s_{5}^{2}= & -\frac{\left(a^{2}+\hat{r}^{2}\right)\left(a^{2}+\hat{r}^{2}-M_{0}\right)}{\Sigma^{2}} d \hat{t}^{2}+\Sigma\left(\frac{\hat{r}^{2} d \hat{r}^{2}}{f^{2}-M_{0} \hat{r}^{2}}+\frac{d \theta^{2}}{4}\right)-\frac{M_{0} F}{\Sigma^{2}}(d \hat{\psi}+\cos \theta d \hat{\phi}) d \hat{t} \\
& +\frac{\Sigma}{4}\left(d \hat{\psi}^{2}+d \hat{\phi}^{2}+2 \cos \theta d \hat{\psi} d \hat{\phi}\right)+\frac{a^{2} M_{0} B}{4 \Sigma^{2}}(d \hat{\psi}+\cos \theta d \hat{\phi})^{2} \\
A= & \frac{M_{0} \sinh 2 \delta}{2 \Sigma}\left(d \hat{t}-\frac{1}{2} a e^{\delta}(d \hat{\psi}+\cos \theta d \hat{\phi})\right)
\end{aligned}
$$

and we defined the quantities

$$
\begin{aligned}
B & =a^{2}+\hat{r}^{2}-2 M_{0} s^{3} c^{3}-M_{0} s^{4}\left(2 s^{2}+3\right), & F & =a\left(\hat{r}^{2}+a^{2}\right)\left(c^{3}+s^{3}\right)-a M_{0} s^{3} \\
\Sigma & =\hat{r}^{2}+a^{2}+M_{0} s^{2}, & f & =\hat{r}^{2}+a^{2},
\end{aligned}
$$


We also note that the $\mathrm{SU}(2)_{L}$ angle $\hat{\psi}$ is identified as

$$
\hat{\psi} \sim \hat{\psi}+4 \pi n .
$$

There is a more general solution with two independent angular momenta, but we have set the other angular momentum, $J_{R}$, to zero. For this case, the $\mathrm{SU}(2)_{R}$ rotational symmetry considerably simplifies matters.

The surface gravities at the inner and outer horizons $r_{ \pm}^{2}=\frac{1}{2}\left(M_{0}-2 a^{2}\right) \pm \frac{1}{2} \sqrt{M_{0}\left(M_{0}-4 a^{2}\right)}$ are

$$
\frac{1}{\kappa_{ \pm}}=\frac{\sqrt{M_{0}}}{2}\left(\left(c^{3}+s^{3}\right) \pm \frac{c^{3}-s^{3}}{\sqrt{1-4 a^{2} / M_{0}}}\right) .
$$

in terms of which the Hawking temperature is $T_{H}=\frac{\kappa_{+}}{2 \pi}$. The horizon angular velocities are

$$
\Omega_{L}=\Omega_{\phi}-\Omega_{\psi}=\frac{4 a}{M_{0}}\left[\left(c^{3}-s^{3}\right)+\left(c^{3}+s^{3}\right) \sqrt{1-4 a^{2} / M_{0}}\right]^{-1}, \quad \Omega_{R}=0,
$$

and the electric potential at the horizon reads

$$
\Phi=\frac{c^{2} s-s^{2} c+\left(c^{2} s+s^{2} c\right) \sqrt{1-4 a^{2} / M_{0}}}{c^{3}-s^{3}+\left(c^{3}+s^{3}\right) \sqrt{1-4 a^{2} / M_{0}}} .
$$

\subsection{Six-dimensional lift}

Any solution of the 5D bosonic minimal supergravity action (3.1) can be lifted (see [40] for details) to a solution of the $6 \mathrm{D}$ theory

$$
S_{6}=\frac{1}{8 \pi^{3}} \int d^{6} x \sqrt{-g}\left(R-\frac{1}{12} H^{2}\right),
$$

where the three-form field strength $H$ is restricted by the self-duality conditions $H=* H$. The lift is given by

$$
\begin{aligned}
d s_{6}^{2} & =d s_{5}^{2}+(d \hat{u}+A)^{2}, \\
H & =-d A \wedge(d \hat{u}+A)-*(d A \wedge(d \hat{u}+A)),
\end{aligned}
$$

where

$$
\hat{u} \sim \hat{u}+2 \pi m
$$

will be referred to as the $\mathrm{U}(1)$ fiber coordinate. The lift of the Kerr-Newman geometry is

$$
\begin{aligned}
d s_{6}^{2}= & -\left(f-M_{0} c^{2}\right) \frac{d \hat{t}^{2}}{\Sigma}+d \hat{u}^{2}+M_{0} \frac{\sinh 2 \delta}{\Sigma} d \hat{t} d \hat{u}+\Sigma\left(\frac{\hat{r}^{2} d \hat{r}^{2}}{f^{2}-M_{0} \hat{r}^{2}}+\frac{d \theta^{2}}{4}\right) \\
& +\frac{\Sigma}{4}\left(d \hat{\psi}^{2}+d \hat{\phi}^{2}+2 \cos \theta d \hat{\phi} d \hat{\psi}\right)+\frac{M_{0} a^{2}}{4 \Sigma}(d \hat{\psi}+\cos \theta d \hat{\phi})^{2} \\
& -\frac{M_{0} a}{\Sigma}\left(\left(c^{3}+s^{3}\right) d \hat{t}+\left(s^{2} c+c^{2} s\right) d \hat{u}\right)(d \hat{\psi}+\cos \theta d \hat{\phi})
\end{aligned}
$$

For many questions, the $6 \mathrm{D}$ picture is simpler than the $5 \mathrm{D}$ one, and we shall specialize to this case when convenient. In particular, the interchange of fiber and angular coordinates which relates black string and black hole descriptions is simply a diffeomorphism when there is a $6 \mathrm{D}$ lift, whereas in the more general context an $\mathrm{M} /$ string duality may be needed to justify the interchange. 


\section{3 $\quad$ Extremality}

We are interested in a family of extremal, non-supersymmetric black holes obtained by setting

$$
M_{0}=4 a^{2} .
$$

In this case

$$
\begin{aligned}
M & =6 a^{2} \cosh 2 \delta \\
J_{L} & =4 a^{3}\left(c^{3}+s^{3}\right), \\
Q & =4 a^{2} s c,
\end{aligned}
$$

the entropy is

$$
S_{B H}=8 \pi a^{3}\left(c^{3}-s^{3}\right)=2 \pi \sqrt{J_{L}^{2}-Q^{3}}
$$

and

$$
\begin{aligned}
\Phi & =\frac{c^{2} s-s^{2} c}{c^{3}-s^{3}} \\
\Omega_{L} & =\frac{1}{a\left(c^{3}-s^{3}\right)} .
\end{aligned}
$$

According to the usual thermodynamic arguments, the quantum state is mixed and given by the density matrix with eigenvalues

$$
e^{-\beta_{H}\left(\omega-m \Omega_{L}+q \Phi\right)}
$$

where $\omega, m$ and $q$ are the energy, $J_{L}$ spin and charge of the state. Determining the density matrix at extremality is slightly subtle because $\beta_{H} \rightarrow \infty$. This implies only states with $\omega=m \Omega_{L}\left(M_{0}=4 a^{2}\right)-q \Phi\left(M_{0}=4 a^{2}\right)$ can contribute. The spin and charge dependence of the remaining density matrix on this subspace is then ${ }^{6}$

$$
e^{-\frac{2 m}{T_{R}}-\frac{3 q}{T_{Q}}}
$$

where

$$
\begin{aligned}
& T_{R}=-2\left[\beta_{H}\left(\Omega_{L}\left(M_{0}\right)-\Omega_{L}\left(4 a^{2}\right)\right)\right]_{M_{0}=4 a^{2}}^{-1}=\frac{c^{3}-s^{3}}{\pi\left(c^{3}+s^{3}\right)}=\frac{\sqrt{1-\frac{Q^{3}}{J_{L}^{2}}}}{\pi} \\
& T_{Q}=3\left[\beta_{H}\left(\Phi\left(M_{0}\right)-\Phi\left(4 a^{2}\right)\right)\right]_{M_{0}=4 a^{2}}^{-1}=\frac{c^{3}-s^{3}}{4 \pi a s^{2} c^{2}}=\frac{\sqrt{J_{L}^{2}-Q^{3}}}{\pi Q^{2}}=\frac{J_{L} T_{R}}{Q^{2}} .
\end{aligned}
$$

The reason for the notation " $T_{R}$ " for the temperature conjugate to angular momentum will become apparent shortly.

\footnotetext{
${ }^{6}$ We have chosen $T_{R}$ to be the variable conjugate to $2 m$, rather than $m$, because for neutral Kerr black holes $m \sim-i \partial_{\hat{\psi}}$ takes half integer values and $\hat{\psi}$ runs from 0 to $4 \pi$. Hence it is $2 m$ which will eventually become, in section 5 , the canonically normalized $L_{0}$ eigenvalue of a $2 \mathrm{D}$ CFT. Similarly there is a factor of 3 in the definition of $T_{Q}$ because $3 q \sim-i \partial_{\hat{u}}$ is an integer.
} 


\subsection{Near-horizon limit}

In this subsection we describe the near-horizon limit, following [18, 39, 41] To reach the near-horizon limit from (3.15), define

$$
\begin{aligned}
& t=\frac{\Omega_{L}}{2 a^{2}} \hat{t} \epsilon, \quad r=\frac{\hat{r}^{2}-a^{2}}{\epsilon}, \quad y=2 \pi T_{Q}(\hat{u}+\Phi \hat{t}), \\
& \psi=\hat{\psi}-\Omega_{L} \hat{t}-\frac{2 J_{L}}{Q^{2}}(\hat{u}+\Phi \hat{t}), \quad \phi=\hat{\phi}
\end{aligned}
$$

The near-horizon metric is

$$
\begin{aligned}
\frac{12}{M} d s^{2}= & -r^{2} d t^{2}+\frac{d r^{2}}{r^{2}}+\gamma(d y+r d t)^{2}+\gamma(d \psi+\cos \theta d \phi)^{2} \\
& +2 \alpha \gamma(d y+r d t)(d \psi+\cos \theta d \phi)+d \theta^{2}+\sin ^{2} \theta d \phi^{2}
\end{aligned}
$$

where the deformation parameters

$$
\begin{aligned}
& \alpha=\frac{2 \cosh 2 \delta}{1+\cosh ^{2} 2 \delta} \\
& \gamma=1+\frac{1}{\cosh ^{2} 2 \delta} .
\end{aligned}
$$

are related by $M \alpha \gamma=12 a^{2}$.

In terms of the $\mathrm{SL}(2, \mathbb{R})_{L} \times \mathrm{SU}(2)_{R}$ invariant forms,

$$
\begin{aligned}
\sigma_{1} & =\cos \psi d \theta+\sin \theta \sin \psi d \phi \\
\sigma_{2} & =-\sin \psi d \theta+\sin \theta \cos \psi d \phi \\
\sigma_{3} & =d \psi+\cos \theta d \phi \\
w_{ \pm} & =-e^{\mp y} r d t \mp e^{\mp y} d r / r \\
w_{3} & =d y+r d t,
\end{aligned}
$$

the metric can be written in the manifestly $\mathrm{SL}(2, \mathbb{R})_{L} \times \mathrm{SU}(2)_{R}$-invariant form

$$
\frac{12}{M} d s^{2}=-w_{+} w_{-}+\gamma w_{3}^{2}+\sigma_{1}^{2}+\sigma_{2}^{2}+\gamma \sigma_{3}^{2}+2 \alpha \gamma w_{3} \sigma_{3} .
$$

The gauge field $A$ is conveniently expressed via

$$
d \hat{u}+A=-\frac{a}{2} \tanh 2 \delta\left(e^{\delta} \sigma_{3}+e^{-\delta} w_{3}\right)
$$

and $H$ is then readily constructed from this expression via (3.13) as

$$
H=\frac{Q}{4}\left(\sigma_{1} \wedge \sigma_{2} \wedge \sigma_{3}+\frac{1}{2} w_{+} \wedge w_{-} \wedge w_{3}+\operatorname{sech} 2 \delta\left(\sigma_{1} \wedge \sigma_{2} \wedge w_{3}+\frac{1}{2} w_{+} \wedge w_{-} \wedge \sigma_{3}\right)\right)
$$

The $(y, \psi)$ identifications are

$$
y \sim y+4 \pi^{2} T_{Q} m, \quad \psi \sim \psi-\frac{4 \pi J_{L} m}{Q^{2}}+4 \pi n,
$$


for any integers $(m, n)$. The $\mathrm{U}(1)$ fiber lies along the vector field

$$
f \equiv \partial_{\hat{u}}=2 \pi T_{Q} \partial_{y}-\frac{2 J_{L}}{Q^{2}} \partial_{\psi}
$$

while gauge-invariant rotations along $J_{L}$ are generated by

$$
j_{L} \equiv \partial_{\hat{\psi}}-A_{\hat{\psi}} f=\frac{2\left(c^{3}-s^{3}\right)}{\sinh 4 \delta}\left(e^{\delta} \partial_{y}-e^{-\delta} \partial_{\psi}\right),
$$

where we have used $A_{\hat{\psi}}=A_{\psi}=-\frac{1}{2} a e^{\delta} \tanh 2 \delta$.

\section{The asymptotic symmetry group}

In this section we briefly review the computation of $[4,12,14,21]$ showing that the asymptotic symmetry group of the near-horizon spacetime (3.26) with boundary conditions which surpress deviations from extremality consists of a centrally-extended Virasoro algebra with central charge $c_{R}=6 J_{L}$. In the convention of this paper, the chiral half of the CFT excited in the case of extreme black holes consists of right-movers. This convention was chosen because this is usually the supersymmetric side and the excitations we consider break supersymmetry.

The near-horizon metric of the $5 \mathrm{~d}$ black hole is

$$
d s_{5}^{2}=\frac{M}{12}\left[-r^{2} d t^{2}+\frac{d r^{2}}{r^{2}}+d \theta^{2}+\sin ^{2} \theta d \phi^{2}+\frac{27 J_{L}^{2}}{M^{3}}\left(d \tilde{y}+\pi T_{R} \cos \theta d \phi+r d t\right)^{2}\right]
$$

where $\tilde{y}=\pi T_{R}\left(\hat{\psi}-\Omega_{L} \hat{t}\right)=y+\pi T_{R} \psi$ is identified as

$$
\tilde{y} \sim \tilde{y}+4 \pi^{2} T_{R} n .
$$

The near-horizon gauge field reads

$$
A=-\frac{1}{2} a e^{\delta} \tanh 2 \delta\left(\frac{d \tilde{y}}{\pi T_{R}}+\cos \theta d \phi+e^{-2 \delta} r d t\right)
$$

The boundary conditions on the deviations $\delta g_{\mu \nu}$ from the background are [21]

$$
\begin{aligned}
& \delta g_{t t} \sim \mathcal{O}\left(r^{2}\right), \quad \delta g_{t r} \sim \mathcal{O}\left(r^{-2}\right), \quad \delta g_{t \theta} \sim \mathcal{O}\left(r^{-1}\right), \quad \delta g_{t \tilde{y}} \sim \mathcal{O}(1), \quad \delta g_{t \phi} \sim \mathcal{O}(r) \\
& \delta g_{r r} \sim \mathcal{O}\left(r^{-3}\right), \quad \delta g_{r \theta} \sim \mathcal{O}\left(r^{-2}\right), \quad \delta g_{r \tilde{y}} \sim \mathcal{O}\left(r^{-1}\right), \quad \delta g_{r \phi} \sim \mathcal{O}\left(r^{-2}\right) \\
& \delta g_{\theta \theta} \sim \delta g_{\theta \tilde{y}} \sim \delta g_{\theta \phi} \sim \mathcal{O}\left(r^{-1}\right), \quad \delta g_{\tilde{y} \tilde{y}} \sim \delta g_{\tilde{y} \phi} \sim \mathcal{O}(1), \quad \delta g_{\phi \phi} \sim \mathcal{O}\left(r^{-1}\right)
\end{aligned}
$$

while boundary conditions on the gauge field $\operatorname{read}^{7}$

$$
\delta A_{t} \sim \mathcal{O}(r), \quad \delta A_{r} \sim \mathcal{O}\left(r^{-2}\right), \quad \delta A_{\theta} \sim \mathcal{O}(1), \quad \delta A_{\tilde{y}} \sim \mathcal{O}\left(r^{-1}\right), \quad \delta A_{\phi} \sim \mathcal{O}\left(r^{-1}\right)
$$

\footnotetext{
${ }^{7}$ The boundary condition on $A_{\tilde{y}}$ can be relaxed to allow for a U(1) current algebra, and we expect it is ultimately necessary to do so. However this current algebra is not the object of our present investigations (or those of [21]) so for simplicity we will continue to suppress it with these slightly stronger boundary conditions.
} 
These boundary conditions lead to an asymptotic symmetry group consisting of a Virasoro algebra generated by the following diffeomorphisms and gauge transformations

$$
\xi(\epsilon)=\epsilon(\tilde{y}) \partial_{\tilde{y}}-\partial_{\tilde{y}} \epsilon(\tilde{y}) r \partial_{r}+\Theta \epsilon(\tilde{y}) f .
$$

Here

$$
\Theta \equiv \frac{a e^{\delta}}{2 \pi T_{R}} \tanh 2 \delta
$$

is the background value of $-A_{\tilde{y}}$ in (4.3). The last term generates the gauge transformation $\delta A=-\Theta d \epsilon(\tilde{y})$. We note that for constant $\epsilon$ we recover the gauge invariant rotation generator $(3.33)$

$$
\xi\left(\pi T_{R}\right)=j_{L}
$$

Given the identification (4.2), the zero mode of $\xi$ is then twice the total angular momentum in the NHEK region. Note that this is not the same as the angular momentum $J_{L}$ of the asymptotically flat black hole, as the latter receives contributions from outside the NHEK region. ${ }^{8}$

The Lie bracket algebra is

$$
\left\{\xi(\epsilon), \xi\left(\epsilon^{\prime}\right)\right\}_{L . B .}=\xi\left(\epsilon \partial_{\tilde{y}} \epsilon^{\prime}-\epsilon^{\prime} \partial_{\tilde{y}} \epsilon\right)
$$

The Dirac bracket algebra of the associated generators is

$$
\left\{Q(\xi), Q\left(\xi^{\prime}\right)\right\}_{D . B .}=Q_{\left\{\xi, \xi^{\prime}\right\}_{L . B .}}+\int k_{\xi}\left(\mathcal{L}_{\xi^{\prime}} g, g\right)
$$

where the expression for $k_{\xi}$ can be found in [43, 44]. This last term gives a central extension of the Virasoro algebra with

$$
c_{R}=6 J_{L}
$$

Using this value of $c_{R}$ and the temperature $T_{R}$ conjugate to angular momentum (3.23), the microscopic Cardy formula then reproduces the macroscopic area law

$$
S_{\text {micro }}=\frac{\pi^{2} c_{R} T_{R}}{3}=2 \pi \sqrt{J_{L}^{2}-Q^{3}}=S_{B H} .
$$

\section{CFT interpretation}

Now we wish to describe the holographic duals of the geometries (3.26). We begin with the maximal case, where Kerr/CFT reduces to AdS/CFT and well-understood stringy methods can be applied. We will see that AdS/CFT methods can also be easily used to understand the linearized deformations, but more work will be required for the general case.

\footnotetext{
${ }^{8}$ Indeed, for the supersymmetric BMPV case, all of the angular momentum is stored outside the nearhorizon region [42].
} 


\subsection{The maximal case}

For $\delta \rightarrow \infty$ with fixed $J_{L}, a \rightarrow P e^{-\delta}, Q^{3} \rightarrow J_{L}^{2}=P^{6}$, and we have a maximally charged 5D Kerr-Newman black hole. This solution can also be reached by increasing the spin of the supersymmetric BMPV black hole up to its maximal value, and was studied as such in $[36,45,46]$. In the limit $\delta \rightarrow \infty$,

$$
\alpha \rightarrow 0, \quad \gamma \rightarrow 1
$$

The last term in (3.28) vanishes and we recover locally $\mathrm{AdS}_{3} \times S^{3}$ as the total space of the $\mathrm{U}(1)$ bundle over the $A d S_{2} \times S^{3} 5 \mathrm{D}$ black hole.

The generators of the enhanced local $\mathrm{SL}(2, \mathbb{R})_{R}$ which appears at maximality are

$$
\begin{gathered}
\bar{H}_{n}=f_{n}(y) \partial_{y}-r f_{n}^{\prime}(y) \partial_{r}-\frac{1}{r} f_{n}^{\prime \prime}(y) \partial_{t} \\
f_{n}(y)=e^{-n y}, \quad n=0, \pm 1
\end{gathered}
$$

while the global $\mathrm{SL}(2, \mathbb{R})_{L}$ generators are

$$
\begin{gathered}
H_{n}=-\left(g_{n}(t)+\frac{1}{2 r^{2}} g_{n}^{\prime \prime}(t)\right) \partial_{t}+r g_{n}^{\prime}(t) \partial_{r}+\frac{1}{r} g_{n}^{\prime \prime}(t) \partial_{y} \\
g_{n}(t)=t^{n+1}, \quad n=0, \pm 1
\end{gathered}
$$

There is also a local enhanced $\mathrm{SU}(2)_{L}$

$$
\begin{aligned}
J^{1} & =\cos \psi \partial_{\theta}+\frac{\sin \psi}{\sin \theta} \partial_{\phi}-\sin \psi \cot \theta \partial_{\psi} \\
J^{2} & =-\sin \psi \partial_{\theta}+\frac{\cos \psi}{\sin \theta} \partial_{\phi}-\cos \psi \cot \theta \partial_{\psi} \\
J^{3} & =\partial_{\psi} .
\end{aligned}
$$

The global $\mathrm{SU}(2)_{R}$ generators are given by a similar expression with $\psi \leftrightarrow \phi$.

Tn the $\delta \rightarrow \infty$ limit, one finds

$$
T_{Q} \rightarrow \frac{3 e^{-2 \delta}}{\pi P}, \quad T_{R} \rightarrow \frac{3 e^{-2 \delta}}{\pi}, \quad f \rightarrow-\frac{2}{P} \partial_{\psi}, \quad j_{L} \rightarrow 3 e^{-2 \delta} \partial_{y}
$$

Note that in this limit the fiber vector $f$ is tangent to the $S^{3}$, while the angular momentum $j$ is tangent to the $\mathrm{AdS}_{3}$ factor! The identification (3.31) becomes

$$
y \sim y, \quad \psi \sim \psi-\frac{4 \pi m}{P}+4 \pi n
$$

Since $P \in \mathbb{Z}$, in the limit taken this way the $4 \pi n$ identification is redundant. The approach to the limit however is subtle and will be discussed in the next subsection.

This geometry also arises as the near horizon of the $P^{3}$ string in $5 \mathrm{D}$, which is locally a U(1) bundle over $A d S_{3} \times S^{2}$. To see this we rewrite the metric at $\delta=\infty$ in the form

$$
d s^{2}=\frac{Q}{4}\left(-w^{+} w^{-}+w_{3}^{2}+d \theta^{2}+\sin ^{2} \theta d \phi^{2}\right)+(d u+\mathcal{A})^{2},
$$


where $\mathcal{A}=-\frac{P}{2} \cos \theta d \phi$ and we have defined a canonically identified coordinate

$$
u=-\frac{P}{2} \psi \sim u+2 \pi m
$$

This is manifestly a $\mathrm{U}(1)$ bundle parameterized by $u$ over $A d S_{3} \times S^{2}$. Integrating the magnetic graviphoton field strength $\mathcal{F}=d \mathcal{A}$ over the $S^{2}$ we see that this corresponds to a magnetic string solution with charge $P$. The holographic dual of this geometry is of course the $P^{3} \mathrm{CFT}$, which therefore must also describe maximal extreme Kerr-Newman.

In $A d S_{3}$, the $\mathrm{SL}(2, \mathbb{R})_{R}$ isometry algebra (5.2) is enhanced to a Virasoro which in the geometry above has $c_{R}=3 \ell / 2 G_{3}=6 P^{3}$. With the boundary conditions of [47] these are generated by the vector fields

$$
\xi_{\text {AdS }}(f)=f(y) \partial_{y}-\partial_{y} f(y) r \partial_{r}+\mathcal{O}\left(r^{-1}\right) \partial_{t}+\mathcal{O}\left(r^{-1}\right) \partial_{y}+\mathcal{O}(1) \partial_{r},
$$

where the correction terms are trivial. ${ }^{9}$ One expects this Virasoro to be directly related to some limit of the Kerr-CFT Virasoro. Indeed, transforming the generators of the latter, given in $(4.5)$, to $(y, \psi)$ coordinates

$$
\begin{aligned}
\xi(\epsilon) & =\epsilon(\tilde{y})\left(\partial_{\tilde{y}}+\Theta f\right)-\partial_{\tilde{y}} \epsilon(\tilde{y}) r \partial_{r} \\
& =\epsilon\left(y+\pi T_{R} \psi\right)\left(\frac{1-2 \pi T_{Q} \Theta}{\pi T_{R}} \partial_{\psi}+2 \pi T_{Q} \Theta \partial_{y}\right)-\partial_{y} \epsilon\left(y+\pi T_{R} \psi\right) r \partial_{r},
\end{aligned}
$$

and taking

$$
\epsilon(x)=f(x)
$$

one finds

$$
\lim _{T_{R} \rightarrow 0} \xi(\epsilon)=\xi_{\text {AdS }}(f) .
$$

Hence we conclude that the standard right-moving Virasoro of $A d S_{3}$ coincides in the maximal limit with the Kerr-CFT Virasoro.

\subsection{Explicit brane construction}

The discussions of the previous sections pertain to essentially any $\mathrm{M} /$ string compactification with a consistent truncation to 5D minimal supergravity. Although the dual CFT will always have central charge $6 P^{3}$ (to leading order), the details of the CFT will depend on the compactification. In this section we give an explicit example of a brane construction.

The $6 \mathrm{D}$ metric (5.7) can be uplifted to a solution of type IIB string theory by interpreting (3.30) as the RR three form field strength and adding a $T^{4}$ of unit radius with coordinates $\left(x^{6}, x^{7}, x^{8}, x^{9}\right)$ to the geometry. This is the near horizon geometry of a certain brane configuration in the compactification to $5 \mathrm{D}$ on $\left(u, x^{6}, x^{7}, x^{8}, x^{9}\right)$ (i.e. $T^{4}$ and the Hopf fiber of the $\left.S^{3} / Z_{P}\right)$. The numbers of branes of each type can be determined by computing the charges. The number of D5 branes wrapping the $T^{4}$ is

$$
Q_{5}=\frac{1}{4 \pi^{2}} \int_{S^{3}} H=P
$$

\footnotetext{
${ }^{9}$ The boundary conditions of [47] are more relaxed than the original ones of [3], but nevertheless consistently lead to the same ASG and central charge. Note that the $r$ coordinate here is not the same as the one of [47].
} 
The number of D1 branes dissolved in the D5 branes is

$$
Q_{1}=\frac{1}{(2 \pi)^{6}} \int_{S^{3} \times T^{4}} \star H=P .
$$

In addition, the fact that the $S^{1}$ parametrized by $u=-\frac{1}{2} P \psi$ is fibered over the $S^{2}$ implies there is a charge $Q_{T N}=P$ magnetic Taub-NUT string

$$
Q_{T N}=\frac{1}{2 \pi} \int_{S^{2}} \mathcal{F}=P
$$

Therefore, the brane configuration that produces the geometry at maximality consists of $P$ copies of

\begin{tabular}{c|cccccc} 
& 6 & 7 & 8 & 9 & $u$ & $\mathbb{R}_{y}$ \\
\hline D5 & $\mathrm{X}$ & $\mathrm{X}$ & $\mathrm{X}$ & $\mathrm{X}$ & & $\mathrm{X}$ \\
$\mathrm{D} 1$ & & & & & & $\mathrm{X}$ \\
$\mathrm{TN}$ & & & & & & $\mathrm{X}$
\end{tabular}

where $\mathbb{R}_{y}$ denotes the common string direction. This can be dualized to three intersecting stacks of M5 branes in M-theory as follows: first, T-dualize on and $x^{8}, x^{9}$ and $u$, which yields the following configuration in type IIA

\begin{tabular}{c|cccccc} 
& 6 & 7 & 8 & 9 & $u$ & $\mathbb{R}_{y}$ \\
\hline D4 & $\mathrm{X}$ & $\mathrm{X}$ & & & $\mathrm{X}$ & $\mathrm{X}$ \\
D4 & & & $\mathrm{X}$ & $\mathrm{X}$ & $\mathrm{X}$ & $\mathrm{X}$ \\
NS5 & $\mathrm{X}$ & $\mathrm{X}$ & $\mathrm{X}$ & $\mathrm{X}$ & & $\mathrm{X}$
\end{tabular}

Lifting to M-theory with an extra coordinate $x^{10}$, we obtain three intersecting stacks of $P$ M5 branes with a common noncompact direction $y$ :

\begin{tabular}{c|ccccccc} 
& 6 & 7 & 8 & 9 & 10 & $u$ & $\mathbb{R}_{y}$ \\
\hline M5 & $\mathrm{X}$ & $\mathrm{X}$ & & & $\mathrm{X}$ & $\mathrm{X}$ & $\mathrm{X}$ \\
M5 & & & $\mathrm{X}$ & $\mathrm{X}$ & $\mathrm{X}$ & $\mathrm{X}$ & $\mathrm{X}$ \\
M5 & $\mathrm{X}$ & $\mathrm{X}$ & $\mathrm{X}$ & $\mathrm{X}$ & & & $\mathrm{X}$
\end{tabular}

This gives an MSW string for a $T^{6}$ compactification of $M$ theory. The self intersection number of this stack of M5 branes is $6 P^{3}$, giving the central charges [34]

$$
c_{L}=c_{R}=6 P^{3} \text {. }
$$

\subsection{Linear deformations}

As mentioned above, there are some subtleties in the $\delta \rightarrow \infty$ limit. These can be understood by doing an expansion in $\alpha \sim 4 e^{-2 \delta}$ around the maximal solution. Since $\gamma \sim 1+\mathcal{O}\left(e^{-4 \delta}\right)$, at this order the only deformation of the geometry (5.7) is in the gauge field

$$
\mathcal{A} \rightarrow \mathcal{A}-\frac{P}{2} \alpha w_{3}
$$


which leads to a radial electric field $\mathcal{F}_{e l}=2 P e^{-2 \delta} d r \wedge d t$ in addition to the magnetic one. This deformation should have a dual interpretation in the $P^{3} \mathrm{CFT}$, presumably as a source for some CFT operator or deformed state. Since it is a deformation of $A d S_{3}$ at this order, this can be analyzed with standard methods.

In addition, there is a deformation of the identification (3.31). At large $\delta$

$$
y \sim y+\frac{12 \pi m e^{-2 \delta}}{P}, \quad \psi \sim \psi-\frac{4 \pi m}{P}+4 \pi n,
$$

To clarify the structure of the lattice of identifications generated by $(m, n)$ it is convenient to redefine $m=\hat{m}+n P$. To leading order in $e^{-2 \delta},(5.19)$ then becomes

$$
y \sim y+12 \pi n e^{-2 \delta}, \quad \psi \sim \psi-\frac{4 \pi \hat{m}}{P},
$$

The $\psi$ identification was already discussed in the previous subsection and produces the geometry of a charge $P$ magnetic string. What is the meaning of this $y$ identification? In general the quotient of $A d S_{3}$ by the $\mathrm{SL}(2, \mathbb{R})_{R}$ element

$$
e^{4 \pi^{2} i T_{R} \bar{H}_{0}}
$$

is dual to a thermal state in the CFT at dimensionless right-moving temperature $T_{R}$. From (5.2) we see that shifts in $y$ are generated by $\bar{H}_{0}$, and the CFT is therefore at temperature

$$
T_{R} \sim \frac{3 e^{-2 \delta}}{\pi},
$$

This is just the linearization in $e^{-2 \delta}$ of the temperature derived in (3.23) as the thermodynamic conjugate to angular momentum. According to Cardy's formula, the entropy to linear order is

$$
S_{\text {micro }}=\frac{\pi^{2} c_{R} T_{R}}{3} \sim 6 \pi J_{L} e^{-2 \delta} .
$$

This agrees exactly with the linearization of the area law (3.18).

Note that, since the temperature itself is already linear in the deformation, the entropy computation (5.23) does not require any knowledge of the deformation of the CFT induced by the operator dual to (5.18). This will however be required at quadratic and higher order.

\subsection{Finite deformations}

At second order, the electric field strength backreacts on the geometry. This breaks the $\mathrm{SL}(2, \mathbb{R})_{R}$ and the $A d S_{3}$ geometry becomes warped. The finitely deformed geometry (3.28) is a solution of $\mathrm{M} /$ string theory and can be reached by a continuous deformation of the $A d S_{3}$ dual of the $P^{3} \mathrm{CFT}$. Therefore it should have a holographic dual which can itself be reached by a continuous deformation of the $P^{3}$ CFT. The analysis of the asymptotic symmetry group suggests that conformal invariance persists in some form for finite deformations, and that the central charge is undeformed to leading order at large $J_{L}$. It is therefore natural to conjecture that the dual of the finitely deformed geometry (3.28) is simply a finite deformation of the $P^{3}$ CFT. This deformation will involve both the dynamics and 
the (mixed) state itself. We note that the finitely deformed geometry (3.28) cannot be glued on to an asymptotically flat 5D string region ${ }^{10}$ (although of course it can be glued on to an asymptotically flat 5D Kerr-Newman). Since everything has been embedded in string theory, this type of warped deformation should have a sensible holographic dual, but it has not been considered in the literature. Closely related deformations were considered in [40, 48-51], see especially the recent paper [52] in which a worldsheet construction is presented. Clearly, a good understanding of this novel type of deformation from the CFT perspective is highly desirable.

\section{Acknowledgments}

We are grateful to Miranda Cheng, Geoffrey Compère, M. Gaberdiel, Tom Hartman, Josh Lapan, Juan Maldacena, Wei Song, C. Vafa and Xi Yin for useful conversations. We would like to thank the Institut d'Études Scientifiques de Cargèse and the Centro Stefano Franscini in Ascona, Monte Verità, where part of this work was carried out, for providing a productive research environment. This work was supported in part by the Fundamental Laws Initiative at Harvard and DOE grant DE-FGO29ER40654 and by ANR grant 08JCJC-0001-0, and the ERC Starting Independent Researcher Grant 240210 — StringQCD-BH.

\section{A Long vs. short string pictures}

For a given black hole, one expects that many different Virasoro algebras exist, with differing central charges associated with different choices of circle in the geometry. This arises already in the study of superysmmetric black holes in toroidal compactifications of string theory. For example, in the case of the $5 \mathrm{D}$ black hole with $Q_{5}$ NS5-branes, $Q_{1}$ fundamental strings wrapping an $S^{1}$ and carrying momentum $n$, T-duality changes the central charge from $6 Q_{1} Q_{5}$ to $6 n Q_{5}$. Of course the temperature also changes and the entropy is always a U-duality invariant — see e.g. [40]. Similarly, in discussions of Kerr-CFT, when there are multiple angular momenta there are multiple sets of consistent boundary conditions with different central charges and temperatures but the same entropy [14, 15]. When a U(1) charge is present and the fiber is a geometric circle, there are still more possibilities [12].

In the present context, there is an alternate and natural computation of the KerrNewman entropy using the Virasoro symmetry associated to charge circle paramterized by $y$ at fixed $\psi$ in (3.28). The corresponding asymptotic symmetry group analysis then gives $[16,17]$

$$
c^{Q}=6 Q^{2}
$$

From the identification (3.31) the dimensionless temperature is $T_{Q}$. The Cardy formula then gives

$$
S_{B H}=2 \pi^{2} Q^{2} T_{Q}=2 \pi \sqrt{J_{L}^{2}-Q^{3}}
$$

\footnotetext{
${ }^{10}$ It is possible that this geometry can be obtained in a near horizon scaling limit of the general black string geometry in which the asymptotically measured M2 charge goes to infinity.
} 
Let us now try to understand the relation between this $Q$-CFT picture and the Kerr-CFT picture at least near the maximal point. The $Q$-CFT lives on the closed circle parameterized by

$$
y=2 \pi T_{Q} s, \quad 0 \leq s \leq 2 \pi .
$$

The fact that the identification (3.31) also involves a shift of $\psi$ means that there is a finite chemical potential for the charge conjugate to $\psi$, or equivalently $\psi$-twisted boundary conditions. This does not affect the Cardy entropy. On the other hand, the Kerr-CFT lives on the circle

$$
\tilde{y}=2 \pi T_{R} s, \quad 0 \leq s \leq 2 \pi .
$$

The ratio of the two $s$-coefficients is

$$
\frac{T_{R}}{T_{Q}}=\frac{Q^{2}}{J_{L}} \rightarrow P \text { for } \delta \rightarrow \infty
$$

Thus near maximality the Kerr-circle wraps $P$ times around the $Q$-circle and hence is a "long-string" — in the sense of [53] — of the Q-CFT. The corresponding rescaled Virasoro generators are related by

$$
L_{m}^{\text {long }}=P L_{m / P},
$$

for $m$ a multiple of $P$. It follows that

$$
\left[L_{m}^{\text {long }}, L_{n}^{\text {long }}\right]=P^{2}\left[L_{m / P}, L_{n / P}\right]=(m-n) L_{m+n}^{\text {long }}+\frac{c}{12 P}\left(m^{3}-m a\right) \delta_{m+n},
$$

where $a$ depends on the definition of the constant $L_{0}$ shift. Hence

$$
c^{\text {long }}=\frac{c}{P} \text {. }
$$

This is precisely the relation between the Kerr/CFT and $Q$-CFT central charge at maximality. Note that moreover the temperature is rescaled by a factor of $P$ because the long string circle is $P$ times bigger. Hence, exactly as in [53], the product $c T$ appearing in the Cardy entropy is the same in long and short string pictures. Similar conclusions might be reached, pending a better understanding of the deformation, more generally whenever the ratio $\frac{T_{R}}{T_{Q}}=\frac{Q^{2}}{J_{L}}$ is rational.

Open Access. This article is distributed under the terms of the Creative Commons Attribution Noncommercial License which permits any noncommercial use, distribution, and reproduction in any medium, provided the original author(s) and source are credited.

\section{References}

[1] A. Strominger and C. Vafa, Microscopic Origin of the Bekenstein-Hawking Entropy, Phys. Lett. B 379 (1996) 99 [hep-th/9601029] [SPIRES].

[2] A. Strominger, Black hole entropy from near-horizon microstates, JHEP 02 (1998) 009 [hep-th/9712251] [SPIRES]. 
[3] J.D. Brown and M. Henneaux, Central Charges in the Canonical Realization of Asymptotic Symmetries: An Example from Three-Dimensional Gravity,

Commun. Math. Phys. 104 (1986) 207 [SPIRES].

[4] M. Guica, T. Hartman, W. Song and A. Strominger, The Kerr/CFT Correspondence, Phys. Rev. D 80 (2009) 124008 [arXiv:0809.4266] [SPIRES].

[5] S.A. Teukolsky, Perturbations of a rotating black hole. 1. Fundamental equations for gravitational electromagnetic and neutrino field perturbations, Astrophys. J. 185 (1973) 635 [SPIRES].

[6] W.H. Press and S.A. Teukolsky, Perturbations of a Rotating Black Hole. II. Dynamical Stability of the Kerr Metric, Astrophys. J. 185 (1973) 649 [SPIRES].

[7] S.A. Teukolsky and W.H. Press, Perturbations of a rotating black hole. III. Interaction of the hole with gravitational and electromagnetic radiation, Astrophys. J. 193 (1974) 443 [SPIRES].

[8] I. Bredberg, T. Hartman, W. Song and A. Strominger, Black Hole Superradiance From Kerr/CFT, JHEP 04 (2010) 019 [arXiv:0907.3477] [SPIRES].

[9] T. Hartman, W. Song and A. Strominger, Holographic Derivation of Kerr-Newman Scattering Amplitudes for General Charge and Spin, JHEP 03 (2010) 118 [arXiv: 0908.3909] [SPIRES].

[10] A. Castro and F. Larsen, Near Extremal Kerr Entropy from AdS 2 Quantum Gravity, JHEP 12 (2009) 037 [arXiv:0908.1121] [SPIRES].

[11] M. Cvetič and F. Larsen, Greybody Factors and Charges in Kerr/CFT, JHEP 09 (2009) 088 [arXiv:0908.1136] [SPIRES].

[12] T. Hartman, K. Murata, T. Nishioka and A. Strominger, CFT Duals for Extreme Black Holes, JHEP 04 (2009) 019 [arXiv: 0811.4393] [SPIRES].

[13] H. Lü, J. Mei and C.N. Pope, Kerr/CFT Correspondence in Diverse Dimensions, JHEP 04 (2009) 054 [arXiv: 0811.2225] [SPIRES].

[14] T. Azeyanagi, N. Ogawa and S. Terashima, Holographic Duals of Kaluza-Klein Black Holes, JHEP 04 (2009) 061 [arXiv: 0811 .4177] [SPIRES].

[15] D.D.K. Chow, M. Cvetič, H. Lü and C.N. Pope, Extremal Black Hole/CFT Correspondence in (Gauged) Supergravities, Phys. Rev. D 79 (2009) 084018 [arXiv:0812.2918] [SPIRES].

[16] T. Azeyanagi, N. Ogawa and S. Terashima, The Kerr/CFT Correspondence and String Theory, Phys. Rev. D 79 (2009) 106009 [arXiv:0812.4883] [SPIRES].

[17] H. Isono, T.-S. Tai and W.-Y. Wen, Kerr/CFT correspondence and five-dimensional BMPV black holes, Int. J. Mod. Phys. A 24 (2009) 5659 [arXiv:0812.4440] [SPIRES].

[18] C.-M. Chen and J.E. Wang, Holographic Duals of Black Holes in Five-dimensional Minimal Supergravity, Class. Quant. Grav. 27 (2010) 075004 [arXiv: 0901.0538] [SPIRES].

[19] H. Lü, J.-w. Mei, C.N. Pope and J.F. Vazquez-Poritz, Extremal Static AdS Black Hole/CFT Correspondence in Gauged Supergravities, Phys. Lett. B 673 (2009) 77 [arXiv:0901.1677] [SPIRES].

[20] A.J. Amsel, G.T. Horowitz, D. Marolf and M.M. Roberts, No Dynamics in the Extremal Kerr Throat, JHEP 09 (2009) 044 [arXiv:0906.2376] [SPIRES]. 
[21] G. Compere, K. Murata and T. Nishioka, Central Charges in Extreme Black Hole/CFT Correspondence, JHEP 05 (2009) 077 [arXiv: 0902.1001] [SPIRES].

[22] C. Krishnan and S. Kuperstein, A Comment on Kerr-CFT and Wald Entropy, Phys. Lett. B 677 (2009) 326 [arXiv:0903.2169] [SPIRES].

[23] D. Astefanesei and Y.K. Srivastava, CFT Duals for Attractor Horizons, Nucl. Phys. B 822 (2009) 283 [arXiv:0902.4033] [SPIRES].

[24] W.-Y. Wen, Holographic descriptions of (near-)extremal black holes in five dimensional minimal supergravity, arXiv:0903.4030 [SPIRES].

[25] T. Azeyanagi, G. Compere, N. Ogawa, Y. Tachikawa and S. Terashima, Higher-Derivative Corrections to the Asymptotic Virasoro Symmetry of $4 d$ Extremal Black Holes, Prog. Theor. Phys. 122 (2009) 355 [arXiv:0903.4176] [SPIRES].

[26] J.-J. Peng and S.-Q. Wu, Extremal Kerr/CFT correspondence of five-dimensional rotating (charged) black holes with squashed horizons, Nucl. Phys. B 828 (2010) 273 [arXiv:0911.5070] [SPIRES].

[27] Y. Matsuo, T. Tsukioka and C.-M. Yoo, Another Realization of Kerr/CFT Correspondence, Nucl. Phys. B 825 (2010) 231 [arXiv:0907.0303] [SPIRES].

[28] Y. Matsuo, T. Tsukioka and C.-M. Yoo, Yet Another Realization of Kerr/CFT Correspondence, Europhys. Lett. 89 (2010) 60001 [arXiv:0907.4272] [SPIRES].

[29] B. Chen and C.-S. Chu, Real-time correlators in Kerr/CFT correspondence, JHEP 05 (2010) 004 [arXiv: 1001.3208] [SPIRES].

[30] X.-N. Wu and Y. Tian, Extremal Isolated Horizon/CFT Correspondence, Phys. Rev. D 80 (2009) 024014 [arXiv:0904.1554] [SPIRES].

[31] M. Becker, S. Cremonini and W. Schulgin, Extremal Three-point Correlators in Kerr/CFT, arXiv: 1004.1174 [SPIRES].

[32] A.J. Amsel, D. Marolf and M.M. Roberts, On the Stress Tensor of Kerr/CFT, JHEP 10 (2009) 021 [arXiv:0907.5023] [SPIRES].

[33] O.J.C. Dias, H.S. Reall and J.E. Santos, Kerr-CFT and gravitational perturbations, JHEP 08 (2009) 101 [arXiv:0906.2380] [SPIRES].

[34] J.M. Maldacena, A. Strominger and E. Witten, Black hole entropy in M-theory, JHEP 12 (1997) 002 [hep-th/9711053] [SPIRES].

[35] N. Alonso-Alberca, E. Lozano-Tellechea and T. Ortín, The near-horizon limit of the extreme rotating $D=5$ black hole as a homogeneous spacetime, Class. Quant. Grav. 20 (2003) 423 [hep-th/0209069] [SPIRES].

[36] M. Guica and A. Strominger, Wrapped M2/M5 duality, JHEP 10 (2009) 036 [hep-th/0701011] [SPIRES].

[37] J.C. Breckenridge et al., Macroscopic and Microscopic Entropy of Near-Extremal Spinning Black Holes, Phys. Lett. B 381 (1996) 423 [hep-th/9603078] [SPIRES].

[38] M. Cvetič and D. Youm, General Rotating Five Dimensional Black Holes of Toroidally Compactified Heterotic String, Nucl. Phys. B 476 (1996) 118 [hep-th/9603100] [SPIRES].

[39] O.J.C. Dias, R. Emparan and A. Maccarrone, Microscopic Theory of Black Hole Superradiance, Phys. Rev. D 77 (2008) 064018 [arXiv:0712.0791] [SPIRES]. 
[40] M.J. Duff, H. Lü and C.N. Pope, $A d S_{3} \times S^{3}$ (un)twisted and squashed and an O(2,2,Z) multiplet of dyonic strings, Nucl. Phys. B 544 (1999) 145 [hep-th/9807173] [SPIRES].

[41] J.M. Bardeen and G.T. Horowitz, The extreme Kerr throat geometry: A vacuum analog of $A d S_{2} \times S^{2}$, Phys. Rev. D 60 (1999) 104030 [hep-th/9905099] [SPIRES].

[42] J.P. Gauntlett, R.C. Myers and P.K. Townsend, Black holes of D $=5$ supergravity, Class. Quant. Grav. 16 (1999) 1 [hep-th/9810204] [SPIRES].

[43] G. Barnich and F. Brandt, Covariant theory of asymptotic symmetries, conservation laws and central charges, Nucl. Phys. B 633 (2002) 3 [hep-th/0111246] [SPIRES].

[44] G. Barnich and G. Compere, Surface charge algebra in gauge theories and thermodynamic integrability, J. Math. Phys. 49 (2008) 042901 [arXiv:0708.2378] [SPIRES].

[45] G.W. Gibbons and C.A.R. Herdeiro, Supersymmetric rotating black holes and causality violation, Class. Quant. Grav. 16 (1999) 3619 [hep-th/9906098] [SPIRES].

[46] L. Dyson, Studies of the over-rotating BMPV solution, JHEP 01 (2007) 008 [hep-th/0608137] [SPIRES].

[47] A.P. Porfyriadis and F. Wilczek, Effective Action, Boundary Conditions and Virasoro Algebra for $A d S_{3}$, arXiv:1007.1031 [SPIRES].

[48] D. Israel, Quantization of heterotic strings in a Goedel/anti de Sitter spacetime and chronology protection, JHEP 01 (2004) 042 [hep-th/0310158] [SPIRES].

[49] D. Israel, C. Kounnas, D. Orlando and P.M. Petropoulos, Electric / magnetic deformations of $S^{3}$ and $A d S_{3}$ and geometric cosets, Fortsch. Phys. 53 (2005) 73 [hep-th/0405213] [SPIRES].

[50] S. Detournay, D. Orlando, P.M. Petropoulos and P. Spindel, Three-dimensional black holes from deformed anti de Sitter, JHEP 07 (2005) 072 [hep-th/0504231] [SPIRES].

[51] D. Anninos, Hopfing and Puffing Warped Anti-de Sitter Space, JHEP 09 (2009) 075 [arXiv: 0809.2433] [SPIRES].

[52] S. Detournay, D. Israel, J.M. Lapan and M. Romo, String Theory on Warped AdS $S_{3}$ and Virasoro Resonances, JHEP 01 (2011) 030 [arXiv: 1007.2781] [SPIRES].

[53] J.M. Maldacena and L. Susskind, D-branes and Fat Black Holes, Nucl. Phys. B 475 (1996) 679 [hep-th/9604042] [SPIRES]. 